\title{
Comparison of Noninvasive Cardiac Test Strategies for Newly Diagnosed Chagas Disease in a Non-Endemic Zone
}

\author{
Elena Refoyo, ${ }^{1,2 *}$ Jesús Troya,${ }^{3}$ Elena Trigo, ${ }^{4}$ Gabriela Guzmán-Martinez, ${ }^{1}$ Silvia Valbuena-López, ${ }^{1}$ Juan Caro-Codón, ${ }^{1}$ \\ Sandra Rosillo, ${ }^{1}$ Mar Moreno-Yanguela, ${ }^{1}$ Juan Tamargo, ${ }^{5}$ José Ramón Arribas, ${ }^{4}$ Harry Acquatella, ${ }^{6}$ and José Lopez-Sendon ${ }^{1}$ \\ ${ }^{1}$ Department of Cardiology, La Paz University Hospital, Autónoma University of Madrid, Madrid, Spain; ${ }^{2}$ Department of Cardiology, Navarra \\ University Clinic, Madrid, Spain; ${ }^{3}$ Department of Internal Medicine, Infanta Leonor University Hospital, Complutense University of Madrid, Madrid, \\ Spain; ${ }^{4}$ Tropical Medicine and Travel Health Unit, Department of Internal Medicine, La Paz University Hospital, Autónoma University of Madrid, \\ Madrid, Spain; ${ }^{5}$ Department of Pharmacology, School of Medicine, Complutense University of Madrid, Madrid, Spain; ${ }^{6}$ School of Medicine,
} University of Caracas, Caracas, Venezuela

\begin{abstract}
Chagas disease is an emerging infectious disease in Europe and other non-endemic areas, mainly owing to migration from endemic areas. We aimed at investigating the value of advanced echocardiography (ECHO) and cardiac magnetic resonance (CMR) in patients newly diagnosed with Chagas disease to compare findings with those of electrocardiogram (ECG) and conventional ECHO and thus detecting cardiac abnormalities. We included consecutive patients with newly diagnosed Chagas disease and registered cardiac test results (ECG, ECHO, and CMR). We divided $\mathrm{ECHO}$ parameters into three tiers: 1) left ventricular ejection fraction, regional wall motion abnormality, and left ventricular diastolic dimension (ECHO-1); 2) other common ECHO parameters (ECHO-2); and 3) global longitudinal strain (GLS) (ECHO-3). Cardiac magnetic resonance included global and segmental biventricular function, the presence of myocardial fibrosis, and edema. The study comprised 100 patients from South America. The mean age was $43.9 \pm 0.9$ years, and $66 \%$ were women. Mean time living in Spain was $9.7 \pm 0.5$ years. The ECG revealed $\geq 2$ abnormal findings in $47 \%$ of patients. $\mathrm{ECHO}-1$ was abnormal in $22 \%$ of patients, $\mathrm{ECHO}-2$ in $52 \%$, and GLS in 16\%. Cardiac magnetic resonance was abnormal in $50 \%$ of cases, and in $3 \%$ of these, ECHO was normal. When ECG and conventional ECHO were taken together, abnormalities were detected in $83 \%$ of patients. This value increased to $86 \%$ and $92 \%$ for GLS and CMR, respectively. These findings suggest that ECG and conventional ECHO should be used routinely as standard cardiac tests for newly diagnosed cases of Chagas disease. The value of advanced ECHO techniques and CMR is low.
\end{abstract}

\section{INTRODUCTION}

Chagas disease, also known as American trypanosomiasis, is included in the WHO list of neglected tropical diseases. ${ }^{1}$ Chagas disease is an emerging infectious disease in Europe and other non-endemic countries, mainly owing to migration from endemic areas of South America. ${ }^{2-4}$ About 10-12 million people could be infected around the world, with an estimated rate of underdiagnosed cases of over $90 \%{ }^{3}$

The natural history of the disease is characterized by two well-established phases. The acute phase (4-8 weeks) is usually oligosymptomatic, with the disease being diagnosed in this phase in only $1-2 \%$ of cases. Mortality is around $1 \%$ (usually related to severe myocarditis or meningoencephalitis). The chronic phase has two distinct clinical forms, namely, the indeterminate form (4-10 weeks after infection), which is characterized by a lack of clinical, radiological, and electrocardiographic manifestations, and the chronic cardiac form, which is defined based on abnormal electrocardiographic findings.

Chagas cardiomyopathy (CCM) constitutes the most serious complication of the disease, affecting $20-40 \%$ of individuals with positive serology results. Chagas cardiomyopathy causes substantial disability and early mortality, especially among young people.

Prompt and correct diagnosis of Chagas disease requires specialized clinical expertise and innovative and intensified disease management. ${ }^{5}$ Traditional diagnosis of chronic CCM has been based on ECG, chest X-ray, and echocardiography $(\mathrm{ECHO})^{6,7}$

*Address correspondence to Elena Refoyo, Hospital Universitario La Paz, Paseo de la Castellana 261, Madrid 28046, Spain. E-mail: erefoyo@gmail.com.
The objectives of this prospective study were 1) to estimate the prevalence of anatomical and functional cardiac abnormalities in recently diagnosed patients before starting etiological treatment and 2) to evaluate the value of traditional cardiac tests and assess the usefulness of cardiac magnetic resonance $(\mathrm{CMR})$ for detecting cardiac involvement in patients with Chagas disease compared with ECG and ECHO (either conventional or advanced).

\section{METHODS}

Study design. We performed an observational, prospective cohort study in La Paz University Hospital (Department of Cardiology and Tropical Diseases Unit) from January 2015 to January 2017.

Patient selection. The initial sample comprised patients older than 18 years with a confirmed diagnosis of Chagas disease according to the $\mathrm{WHO}$ criteria $^{3}$ and attended at the Tropical Diseases Unit. We selected 175 patients with positive values in both the immunofluorescence antibody test (over 1/32) and an ELISA index $>1.1$. Because of exclusion criteria (pregnancy, previous treatment for Chagas disease, alcohol or drug abuse, advanced renal failure [glomerular filtration rate $<30 \mathrm{~mL} /$ minute], previous coronary or cerebrovascular disease, active nonChagas infection, another cardiomyopathy, and general contraindication for CMR imaging), we excluded 75 patients, We finally enrolled 100 consecutive patients in the registry following a predefined protocol. Study data were collected and managed using the REDCap ${ }^{\circledR}$ electronic data capture tools ${ }^{8}$ and hosted at the IdiPAZ Research Institute, Madrid, Spain.

Diagnostic methods. All patients underwent a physical examination, ECG, conventional Doppler 2D-ECHO (ECHO) plus speckle tracking of strain, and CMR. ECG and transthoracic $\mathrm{ECHO}$ were performed on the same day as the clinical 
examination by a board-certified cardiologist. Cardiac magnetic resonance was performed within 60 days of the $\mathrm{ECHO}$.

The ECG result was considered abnormal and suggestive of cardiac involvement if the test revealed two alterations (BENEFIT study). This approach was adopted to ensure that we had a higher risk population. ${ }^{6}$

Echocardiogram. All patients underwent standard transthoracic ECHO examinations with the Philips iE33 transducer X5-1 according to the recommendations of the American Society of Echocardiography (ASE) and the European Association of Cardiovascular Imaging. ${ }^{4,9,10}$ Average longitudinal strain was semiautomatically calculated using QLAB 10 3DQA software application (Advanced Cardiac Quantification 3D Philips Healthcare, Andover, MA).

We divided ECHO parameters into three tiers: $\mathrm{ECHO}-1$ (used in the BENEFIT study as markers of cardiac involvement), that is, left ventricular dilatation $>55 \mathrm{~mm}$, left ventricular ejection fraction (LVEF) $<50 \%$ or regional wall motion abnormalities ${ }^{6}$; ECHO-2, that is, other abnormal 2D-ECHO parameters; and ECHO-3, that is, advanced ECHO, global longitudinal strain (GLS) > -20.

Cardiac magnetic resonance was performed using a 1.5-T scanner (Siemens Avanto DOT, Erlangen, Germany). The use of non-contrast T2-weighted sequences (T2-short tau inversion recovery [STIR]) enabled evaluation of myocardial edema, defined as a regional myocardial increased T2 signal compared with adjacent skeletal muscle. A TrueFISP late gadolinium enhancement (LGE) sequence was used 10-12 minutes after contrast administration to evaluate LGE. Late gadolinium enhancement was defined by increased signal intensity above apparent normal myocardium from 2 to 6 SDs. All CMR analyses were performed using the Siemens Syngo Via VB10A_HF04 application (Siemens AG, Munich, Germany). In cases of ventricular aneurysm and subendocardial or transmural delayed enhancement pattern on CMR, computed tomography (CT) angiography was performed to exclude the possibility of ischemic heart disease.

Patients were classified according to the recommendations of the Society for Cardiovascular Magnetic Resonance and the ASE/InterAmerican Association of Echocardiography (ECOSIAC)/Cardiovascular Imaging Department of the Brazilian Society of Cardiology (DIC-SBC) $)^{4,11,12}$ as follows: stage $A$, that is, the indeterminate form (positive serology with normal ECG, no clinical heart failure, and no heart disease in the $\mathrm{ECHO}$ ); stage $B 1$, that is, asymptomatic patients with structural cardiomyopathy and abnormal ECG or ECHO but normal left ventricle (LV) function; stage B2, that is, impaired LVEF with no registered signs or symptoms of heart failure; stage $C$, that is, LV dysfunction with current or prior heart failure; and stage $D$, that is, patients with symptoms of heart failure at rest, refractory to maximized medical therapy, New York Heart Association functional classification IV, and who require specialized and intensive interventions. ${ }^{13}$

The study protocol (PI-2681) was approved by the Ethics Committee at La Paz University Hospital. All patients signed the informed consent document.

Statistical analysis. The normality of the variables was evaluated using the Levene and the Kolmogorov-Smirnov tests. Normally distributed continuous variables were expressed as mean $\pm S D$, and categorical variables were presented as percentages. A $P$-value $<0.05$ was considered statistically significant. Continuous variables were analyzed using the general linear model. The significance of different time points for each continuous variable was determined using least significant difference analysis.

Non-normally distributed variables are presented as median and interquartile range, and groups were compared using the Wilcoxon rank-sum test. The $\mathrm{X}^{2}$ test or Fisher's exact test was used when appropriate to compare the categorical variables. All analyses were performed using SPSS 21.0 (IBM Corp., Armonk, NY).

\section{RESULTS}

We included 100 consecutive South American patients (66\% women; mean age, $43.9 \pm 0.9$ years). Bolivia was the country of origin of $97 \%$ of cases, and patients had lived in Spain for $9.7 \pm 0.5$ years. A family history of Chagas disease was found in $20 \%$ of cases.

Symptoms were recorded in $58 \%$ of patients, with chest pain and palpitations being the most frequent (Table 1). In the asymptomatic patients, 28 (67\%) presented an abnormal ECG result, 33 (79\%) had ECHO abnormalities, and 22 (52\%) had CMR abnormalities.

Diagnostic methods. The ECG result was normal in 37\% of patients (95\% Cl: $27-47), 16 \%$ presented an abnormality, and $47 \%$ fulfilled the ECG BENEFIT criteria for Chagas cardiac involvement (Table 2, Figure 1). The most frequent abnormalities were right bundle branch block (36\%) and sinus bradycardia below $50 \mathrm{bpm}$ (35\%). No patients were taking concomitant beta-blockers or other drugs that could interfere with cardiac rhythm. Despite normal ECG in $37 \%$ of patients, 9 (24\%) presented ECHO-1, 27 (72\%) ECHO-2, and 18 (48\%) CMR abnormalities.

Echocardiogram. ECHO-1: Twenty-two patients (95\% Cl: 14-30) fulfilled the BENEFIT criteria for cardiac involvement. ECHO-2: In $69 \%$ of patients, other 2D ECHO abnormalities were identified. ECHO-3: $16 \%$ of patients presented a GLS > -20 (Table 2, Figure 1).

Cardiac magnetic resonance. In $50 \%$ of patients $(95 \% \mathrm{Cl}$ : 40-60), CMR revealed at least one abnormality. The most common abnormalities were an increase in left ventricular end-systolic volume, which was higher in men, and alterations of $L V$ regional contractility in the middle and lower apical

\section{TABLE 1}

Main demographic characteristics and clinical symptoms after diagnosis of Chagas disease

\begin{tabular}{lc}
\hline Demographic (mean, SD, \%) & \\
Age (years) & $43.9 \pm 0.9$ \\
Men & $34 / 100(34 \%)$ \\
Residence in Spain (years) & $9.73 \pm 0.47$ \\
Family history of sudden death & $25 / 100(25 \%)$ \\
Family history of Chagas disease & $20 / 100(20 \%)$ \\
Hypertension & $7 / 100(7 \%)$ \\
Diabetes & $5 / 100(5 \%)$ \\
Hypercholesterolemia & $14 / 100(14 \%)$ \\
Symptoms & \\
Global & $58 / 100(58 \%)$ \\
Chest pain & $25 / 100(25 \%)$ \\
Palpitations & $25 / 100(25 \%)$ \\
NYHA & \\
Class II & $15 / 100(15 \%)$ \\
Class III & $1 / 100(1 \%)$ \\
Pre-syncope & $7 / 100(7 \%)$ \\
Syncope & $2 / 100(2 \%)$ \\
Symptomatic heart failure & $16 / 100(16 \%)$ \\
\hline
\end{tabular}


TABLE 2

Cardiac tests after diagnosis of Chagas disease

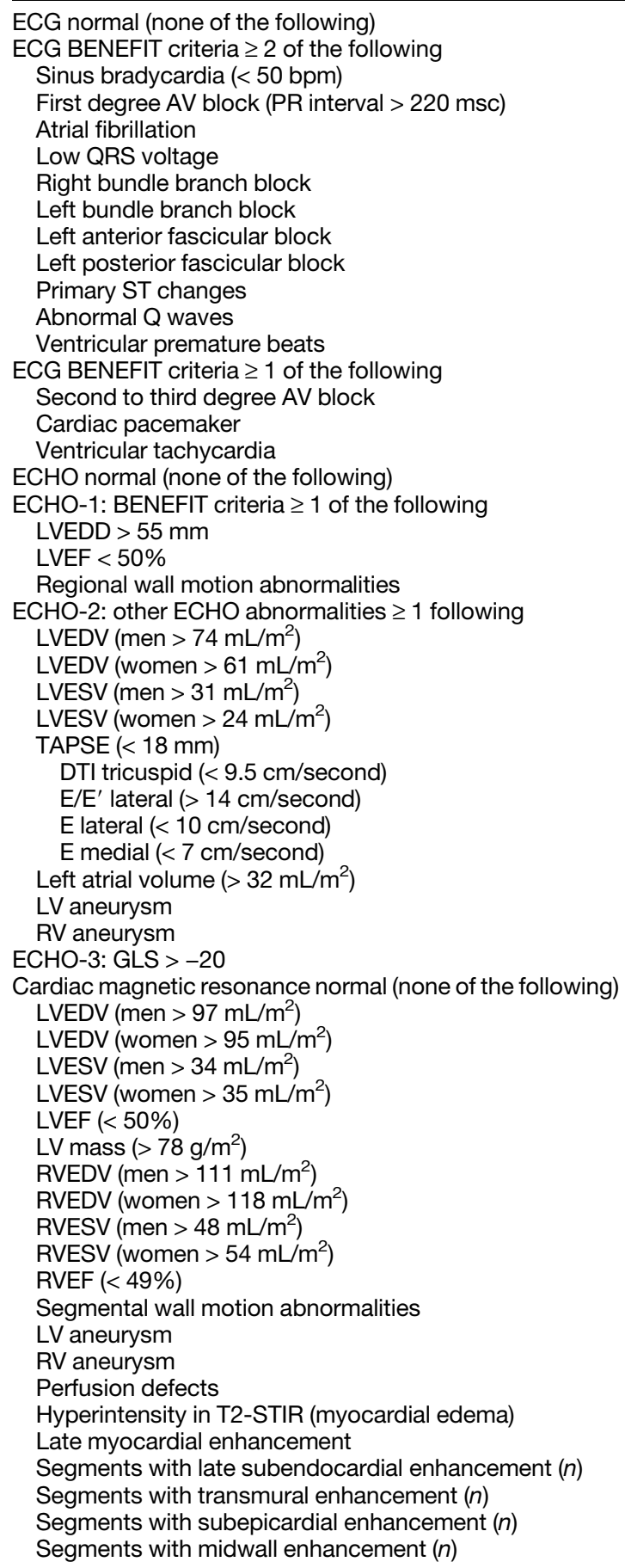

AV = atrioventricular; DTI = Doppler tissue imaging; GLS = global longitudinal strain; LV = left ventricle; LVEDD = left ventricular end diastolic diameter; LVEDV = left ventricular end-diastolic volume; LVEF = left ventricular ejection fraction; LVESV = left ventricular end-systolic volume; $P R=$ time from the onset of the $P$ wave to the start of the QRS complex; $Q R S=$ combination of the $Q$ wave, $\mathrm{R}$ wave and $\mathrm{S}$ wave in ECG; RV = right ventricle; RVEDV = right ventricular end-diastolic volume; RVEF = right ventricular ejection fraction; RVESV = right ventricular end-systolic volume; $\mathrm{ST}$ = segment in ECG that connects the QRS complex and the T wave; TAPSE = tricuspid annular plane systolic excursion.

${ }^{*}$ No patients registered with NYHA Class I or IV.

segments. There was significant concordance between volumes and LVEF measured by ECO and CMR $(r=0.591, P<$ $0.0001)$. Myocardial edema was detected in $16 \%$ of cases $(12 \%$ in the LV, $8 \%$ in the right ventricle, and $4 \%$ in both ventricles). Late gadolinium enhancement (suggestive of myocardial fibrosis) was detected in $15 \%$ of patients. This was heterogeneously distributed, with more frequent intramyocardial and apical and inferior intramyocardial predominance (Table 2, Figure 1).

Computed tomography scans were performed in 13 patients with late enhancement (nine transmural, two subendocardial, and two with a ventricular aneurysm in ECHO and CMR). No significant epicardial coronary artery disease was detected. 


\section{Cardiac abnormalities in Chagas disease}

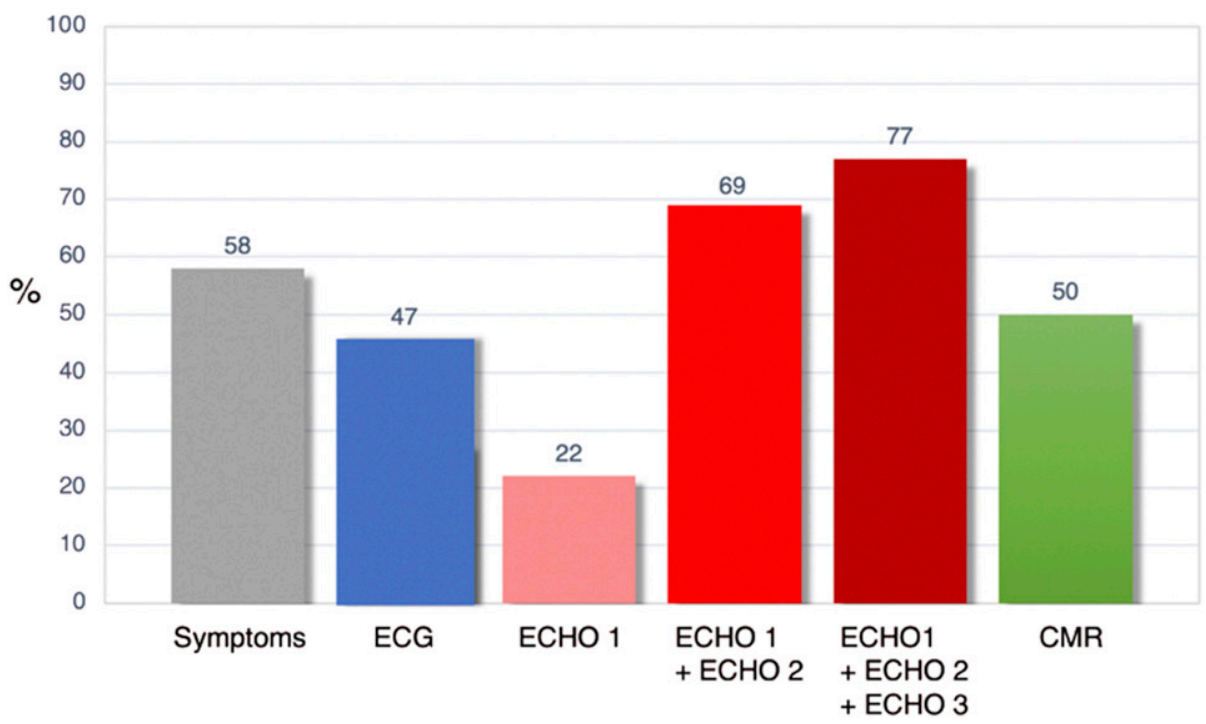

FIGURE 1. Abnormal cardiac findings using different diagnostic techniques. ECG: BENEFIT criteria; Echocardiography (ECHO)-1: BENEFIT criteria; ECHO-2: any other conventional 2D-echo Doppler abnormal finding; ECHO-3: abnormal global longitudinal strain; cardiac magnetic resonance (CMR): any abnormal finding in CMR imaging. This figure appears in color at www.ajtmh.org.

Combined use of diagnostic methods. At least one abnormality was detected using several complementary exploratory methods (ECG; ECHO-1, 2, and 3; and CMR) in 92\% of patients. The exploratory combination that most dramatically increased the ability to detect a cardiac abnormality was ECG with a complete conventional 2D-ECHO (ECHO-2). Addition of GLS or CMR slightly increased the number of patients with cardiac abnormalities (Figure 2).

When patients were staged according to ASE/ECOSIAC/ DIC-SBC recommendations, $47 \%$ of patients were classified as stage $A, 37 \%$ as stage $B 1,5 \%$ as stage $C$, and $1 \%$ as stage $\mathrm{C}$ (Table 3). Eleven patients were not included in this classification, as they had normal systolic function despite having symptoms of heart failure.

\section{DISCUSSION}

Despite our initial expectations of finding few cardiac abnormalities in patients newly diagnosed with Chagas disease, $92 \%$ of patients presented abnormalities in ECG, ECHO, or

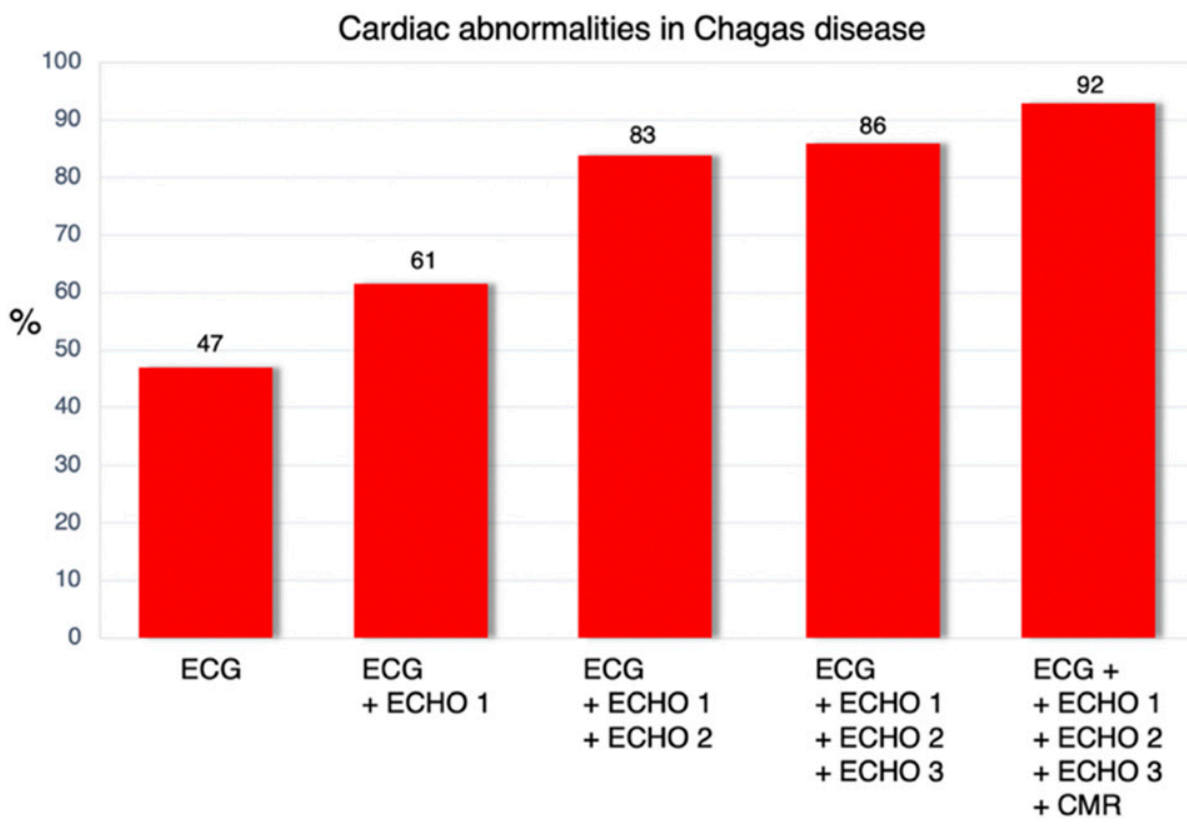

FIGURE 2. Combining diagnostic techniques increased the number of patients presenting some abnormal findings. ECG: BENEFIT criteria. Echocardiography (ECHO)-1: BENEFIT criteria; ECHO-2: any other abnormal finding in conventional 2D-echo Doppler; ECHO-3: abnormal global longitudinal strain; cardiac magnetic resonance (CMR): any abnormal finding in CMR imaging. This figure appears in color at www.ajtmh.org. 
TABLE 3

Number of patients according to the AES/ECOSIAC/DIC-SBC grades/ stages of Chagas cardiomyopathy

\begin{tabular}{lc}
\hline AES Grading & $n(\%)$ \\
\hline ASE/ECOSIAC - A & $47(47 \%)$ \\
ASE/ECOSIAC - B1 & $37(37 \%)$ \\
ASE/ECOSIAC - B2 & 0 \\
ASE/ECOSIAC - C & $5(5 \%)$ \\
ASE/ECOSIAC - D & 0 \\
\hline
\end{tabular}

ASE = American Society of Echocardiography; DIC-SBC = Cardiovascular Imaging Department of the Brazilian Society of Cardiology; ECOSIAC = InterAmerican Association of Echocardiography.

CMR. This finding contrasts with the findings of previous studies, where the prevalence of CCM was lower, ranging from $20 \%$ to $30 \% .^{4}$ There are several explanations for this. First, in the other studies, prevalence was based on clinical ECG and X-ray results, which are less sensitive than ECHO and CMR. Second, more than half of the patients in our study were symptomatic at diagnosis and had probably been infected many years before traveling to Spain from Chagasendemic areas. Furthermore, considering the accepted ECG and $\mathrm{ECHO}$ abnormalities recommended in the BENEFIT trial, ${ }^{6}$ $61 \%$ fulfilled the criteria for Chagas cardiac disease.

ECG abnormalities were more common in our study than those reported in other epidemiological studies, ${ }^{14,15}$ although in many cases, these findings are not specific to Chagas disease. In addition, a normal ECG result is rarely recorded in patients with severe LV dysfunction. ${ }^{4,14,16}$ Despite the low specificity of ECG, it is important to note that this technique is almost universally available.
The basic ECHO criteria recommended in the BENEFIT trial were found in a relatively low number of patients (22\%), with only two patients presenting LV aneurysm. Nevertheless, the frequency of abnormal echocardiograms increased to $74 \%$ when other conventional 2D-ECHO parameters were taken into consideration. Although GLS > -20 , an advanced $\mathrm{ECHO}$ parameter, is related to ventricular arrhythmia, ${ }^{17}$ we only recorded it in three patients. As reported elsewhere, ${ }^{4,18} \mathrm{ECHO}$ abnormalities have been related to various outcomes, including symptomatic heart failure and mortality.

Cardiac magnetic resonance imaging enables better assessment of cardiac anatomy and biventricular function than $\mathrm{ECHO}$, thus making this a very specific technique for appropriately characterizing $\mathrm{CCM} .^{4,19-22}$ The cardiac abnormalities we detected in $50 \%$ of the CMR studies included late myocardial enhancement (suspected of being related to malignant arrhythmias and sudden cardiac death), right ventricular dysfunction, and myocardial edema (suggestive of the persistence of inflammatory processes in the myocardium as described in previous studies). ${ }^{23}$

The addition of conventional echocardiogram to ECG, as in our cohort, significantly increases the number of patients with cardiac abnormalities. Likewise, the use of more advanced and complex ECHO techniques (GLS) or CMR does not significantly increase sensitivity for detecting cardiac abnormalities. In this sense, although advanced ECHO and CMR imaging enable more precise characterization of cardiac involvement in patients with Chagas disease, these techniques are expensive and not available throughout the world. Consequently, they should be reserved for special cases and

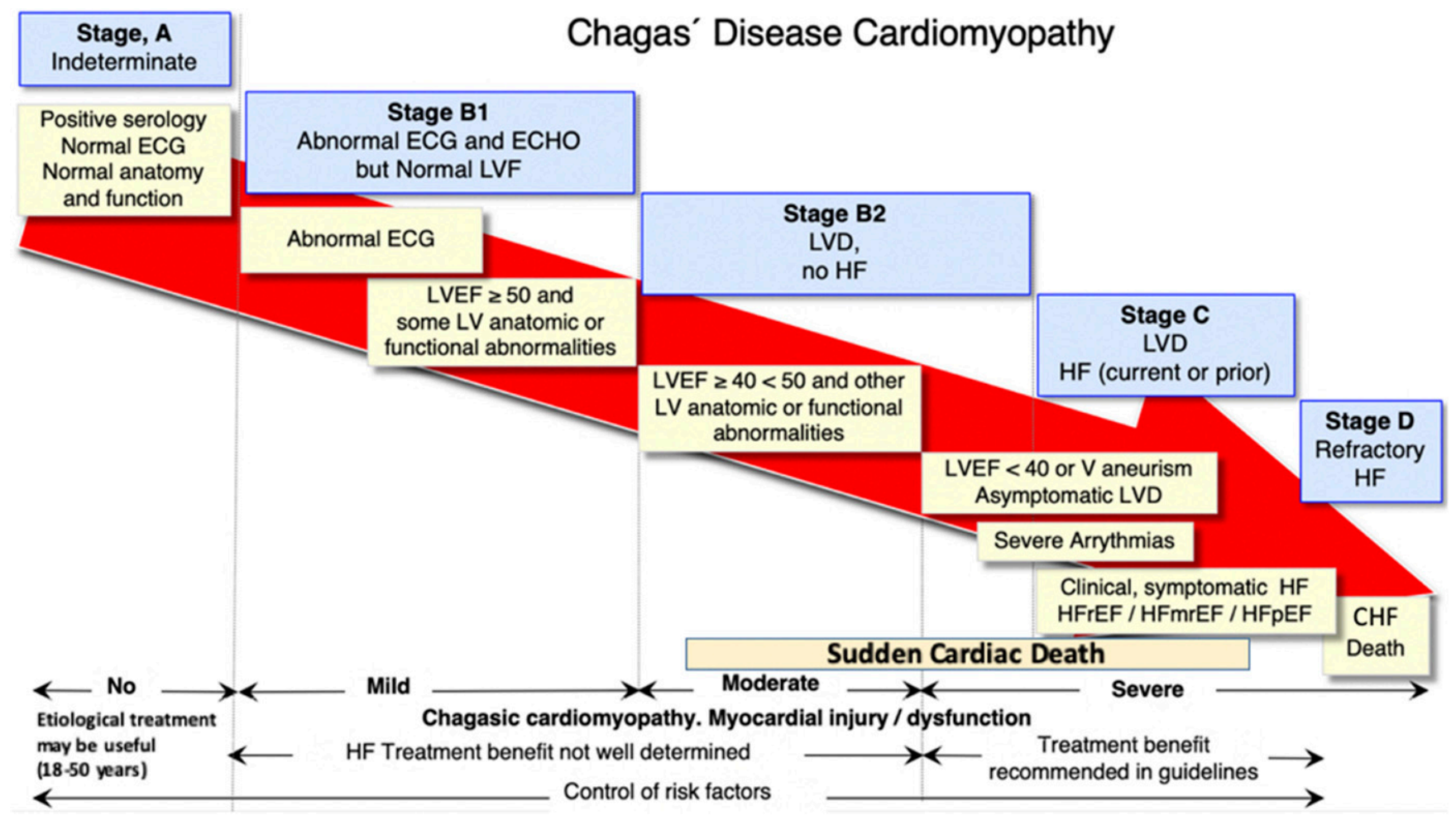

FIGURE 3. Progressive myocardial injury and left ventricular dysfunction and evidence-based treatment recommended in clinical practice guidelines. Both American Society of Echocardiography/InterAmerican Association of Echocardiography stages (blue boxes). $\mathrm{HF}=\mathrm{clinical}$ heart failure; HFrEF/HFmrEF/HFpEF = heart failure with reduced, mid-range; LV = left ventricle; LVD = left ventricular dysfunction; LVF = left ventricular function, and preserved ejection fraction, respectively. This figure appears in color at www.ajtmh.org. 
research purposes. Accordingly, ECG and simple 2D-ECHO imaging should be recommended as standard techniques in all patients newly diagnosed with Chagas disease.

Most patients with abnormal findings were classified as defined by the ASE grading system. Whether treatment for these earlier forms should be initiated remains an important question. In the BENEFIT trial, trypanocidal therapy with benznidazole in patients with established Chagas disease significantly reduced detection of the parasite in serum but failed to improve clinical outcomes. ${ }^{6}$ Although ASE grading shows disease progression, it does not enable identification of groups with asymptomatic LV dysfunction, as previously explored in clinical trials. Therefore, we propose an alternative classification that could better adjust to the recommendations of the European Society of Cardiology and American Heart Association/American College of Cardiology guidelines for the treatment of LV dysfunction and heart failure (Figure 3), namely, recommending treatment only in patients with heart failure or LVEF $<40 \%$. Further clinical research is needed to explore the benefit of treatments in patients with any abnormality of LV dysfunction and preserved LVEF (e.g., > 40\%).

Our study has several limitations that may affect interpretation of the results. First, patient selection was biased, in that we performed the tests in a very specific tropical disease unit, with more than half of the patients symptomatic at diagnosis. Second, the small sample numbers do not enable us to reliably determine the prevalence of less frequent manifestations such as myocardial edema, ventricular tachycardia, complete atrioventricular block, and heart failure. Third, during the study time period, available ECHO software application did not allow us to analyze other speckle-tracking parameters as the global radial or circumferential left ventricular strain or global right ventricular longitudinal strain. Furthermore, sensitive biomarkers of myocardial damage or dysfunction, such as troponins and $\mathrm{N}$-terminal pro brain natriuretic peptide, were not routinely determined in all patients yet may have reinforced the results obtained by ECG and $\mathrm{ECHO}{ }^{24}$

In conclusion, our data show that the prevalence of cardiac involvement in patients newly diagnosed with Chagas disease may range from $61 \%$ to $92 \%$ depending on whether we use the conventional criteria or new imaging techniques such as advanced ECHO and CMR. ECG and conventional ECHO should be recommended as the standard diagnostic techniques in all new cases of Chagas disease. Advanced $\mathrm{ECHO}$ (GLS) and CMR do not significantly increase the number of cases with cardiac abnormalities to justify routine use. Further studies are needed to determine the prognostic value of these findings.

Received April 30, 2020. Accepted for publication June 20, 2020.

Published online July 20, 2020.

Authors' addresses: Elena Refoyo, Gabriela Guzmán-Martinez, Silvia Valbuena-López, Juan Caro-Codón, Sandra Rosillo, Mar MorenoYanguela, and José Lopez-Sendon, Department of Cardiology, Hospital Universitario La Paz, Madrid, Spain, E-mails: erefoyo@gmail.com, gabrielaguzman.ny@hotmail.com, silviacayetana@gmail.com, juancarocd@ gmail.com, sandraofelia.rosillo@salud.madrid.org, marmorenoyanguela@ gmail.com, and jlopezsendon@gmail.com. Jesús Troya, Department of Internal Medicine, Hospital Universitario Infanta Leonor, Madrid, Spain, E-mail: jesus.troya@salud.madrid.org. Elena Trigo and José Ramón Aribas, Infectious Diseases, Hospital Universitario La Paz, Madrid, Spain, E-mails: elenatrigoesteban@hotmail.com and joserarribas@gmail.com. Juan
Tamargo, Department of Pharmacology, Complutense University of Madrid, Madrid, Spain, E-mail: jtamargo@med.ucm.es. Hamy Acquatella, School of Medicine, University Hospital of Caracas, Caracas, Venezuela, E-mail: hacquatella@gmail.com.

\section{REFERENCES}

1. WHO, 2017. Fourth WHO Report on Neglected Tropical Diseases. Integrating Neglected Tropical Diseases into Global Health and Development. Geneva, Switzerland: World Health Organization.

2. Pérez-Molina JA, Molina I, 2018. Chagas disease. Lancet 391: 82-94.

3. WHO, 2018. WHO Report. Chagas Disease. American Trypanosomiasis. Geneva, Switzerland: World Health Organization. Available at: https://www.who.int/chagas/en/. Last consulted July 7, 2019.

4. Acquatella $\mathrm{H}$ et al., 2018. Recommendations for multimodality cardiac imaging in patients with Chagas disease: a report from the American Society of Echocardiography in collaboration with the Inter American Association of Echocardiography (ECOSIAC) and the Cardiovascular Imaging Department of the Brazilian Society of Cardiology (DIC-SBC). J Am Soc Echocardiogr 31: 3-25.

5. Bern C, 2015. Chagas' disease. N Engl J Med 373: 456-466.

6. Marin-Neto JA, Rassi A, Jr., Morillo CA, Avezum A, Connolly SJ, Sosa-Estani S, Rosas F, Yusuf S, BENEFIT Investigators, 2008. Rationale and design of a randomized placebo-controlled trial assessing the effects of etiologic treatment in Chagas' cardiomyopathy: the BENznidazole Evaluation for Interrupting Trypanosomiasis (BENEFIT). Am Heart J 156: 37-43.

7. Morillo CA et al., 2015. Randomized trial of benznidazole for chronic Chagas' cardiomyopathy. N Engl J Med 373: 1295-1306.

8. Harris PA, Taylor R, Thielke R, Payne J, González N, Conde JG, 2009. Research Electronic Data Capture (REDCap) a metadatadriven methodology and workflow process for providing translational research informatics support. J Biomed Inform 42: 377-381.

9. Lang RM et al., 2015. Recommendations for cardiac chamber quantification by echocardiography in adults: an update from the American Society of Echocardiography and the European Association of Cardiovascular Imaging. J Am Soc Echocardiogr 28: 1-39.

10. Galderisi M et al., 2017. Standardization of adult transthoracic echocardiography reporting in agreement with recent chamber quantification, diastolic function, and heart valve disease recommendations: an expert consensus document of the European Association of Cardiovascular Imaging. Eur Heart $J$ Cardiovasc Imag 18: 1301-1310.

11. Kramer KM, Barkhausen J, Flamm SD, Raymond JK, Nagel E, Society for Cardiovascular Magnetic Resonance and Board of Trustees Task Force on Standardized Protocols, 2013. Standardized cardiovascular magnetic resonance (CMR) protocols 2013 update. J Cardiovasc Magn Reson 15: 91.

12. Schulz-Menger J et al., 2013. Standardized image interpretation and post processing in cardiovascular magnetic resonance: Society for Cardiovascular Magnetic Resonance (SCMR) board of trustees task force on standardized post processing. $J$ Cardiovasc Magn Reson 15: 35.

13. Ponikowski P et al.; ESC Scientific Document Group, 2016. Guidelines for the diagnosis and management heart failure: The task force for the diagnosis and treatment of acute and chronic heart failure of the European Society of Cardiology (ESC) developed with the special contribution of the Heart Failure Association (HFA) of the ESC. Eur Heart J 37: 2129-2200.

14. Fernandez $A B$, Nunes $M C$, Clark $E H$, Samuels $A$, Menacho $S$, Gomez J, 2015. Bozo electrocardiographic and echocardiographic abnormalities in Chagas disease: findings in residents of rural Bolivian communities hyperendemic for Chagas disease. Glob Heart 10: 159-166.

15. Marcolino MS, Palhares DM, Ferreira LR, Ribeiro AL, 2015. Electrocardiogram and Chagas disease: a large population database of primary care patients. Glob Heart 10: 167-172. 
16. Rassi A, Jr., Rassi A, Little WC, Xavier SS, Rassi SG, Rassi AG, Rassi GG, Hasslocher-Moreno A, Sousa AS, Scanavacca MI, 2006. Development and validation of a simple risk score for predicting mortality in Chagas' heart disease. $N$ Engl $J$ Med 355: 799-808.

17. Acquatella $\mathrm{H}, 2007$. Echocardiography in Chagas heart disease. Circulation 115: 1124-1131.

18. Acquatella H, Gómez Mancebo J, Catalioti F, Puigbo J, 2013. Imaginología en el diagnóstico y el pronóstico de la enfermedad de Chagas. Rev Argentina Cardiol 81: 184-195.

19. Moreira HT et al., 2017. Evaluation of right ventricular systolic function in Chagas disease using cardiac magnetic resonance imaging. Circ Cardiovas Imag 10: e005571.

20. Rochitte CE et al., 2005. Myocardial delayed enhancement by magnetic resonance imaging in patients with Chagas' disease: a marker of disease severity. J Am Coll Cardiol 46: 1553-1558.
21. Regueiro A, García-Álvarez A, Sitges M, Ortiz-Pérez JT, De Caralt MT, Pinazo MJ, De Caralt MT, Pinazo MJ, Posada E, Heras M, Gascón J, 2013. Myocardial involvement in Chagas disease: insights from cardiac magnetic resonance. Int $J$ Cardiol 165: 107-112.

22. Lee-Felker SSA, Thomas M, Felker ER, Traina M, Salih $M$, Hernandez S, Bradfield J, Lee M, Meymandi S, 2016. Value of cardiac MRI for evaluation of chronic Chagas disease cardiomyopathy. Clin Radiol 71: 618.e1-618.e7.

23. Diaz A et al., 2019. Cardiovascular magnetic resonance imaging evidence of edema in chronic chagasic cardiomyopathy. Cardiol Res Pract 2019: 6420364.

24. Merás $P$, Caro J, Trigo E, Irazusta J, López-Sendón JL, Refoyo E, 2017. Diagnostic value of NT-proBNP for early identification of Chagas cardiomyopathy in non-endemic areas. Rev Esp Cardiol 70: 783-785. 